OPEN ACCESS

Edited by:

Nicole Borel,

University of Zurich, Switzerland

Reviewed by:

Alison Favaroni,

Friedrich-Loeffler-Institut, Germany

Cory Ann Leonard,

East Tennessee State University, United States

*Correspondence:

Kyle H. Ramsey

kramse@midwestern.edu

Specialty section:

This article was submitted to

Infectious Diseases,

a section of the journal

Frontiers in Microbiology

Received: 27 September 2018 Accepted: 17 December 2018

Published: 16 January 2019

Citation:

Gallegos KM, Taylor CR, Rabulinski DJ, Del Toro R, Girgis DE, Jourha D, Tiwari V, Desai UR and Ramsey KH (2019) A Synthetic, Small, Sulfated Agent Is a Promising Inhibitor of Chlamydia spp. Infection in vivo Front. Microbiol. 9:3269. doi: 10.3389/fmicb.2018.03269

\section{A Synthetic, Small, Sulfated Agent Is a Promising Inhibitor of Chlamydia spp. Infection in vivo}

\author{
Karen M. Gallegos ${ }^{1}$, Christopher R. Taylor ${ }^{1,2}$, Daniel J. Rabulinski1, Rosalinda Del Toro', \\ Danielle E. Girgis' ${ }^{1}$, Dapinder Jourha ${ }^{1}$, Vaibhav Tiwari ${ }^{1}$, Umesh R. Desai ${ }^{3}$ and \\ Kyle H. Ramsey ${ }^{*}$ * \\ ${ }^{1}$ Department of Microbiology and Immunology, College of Graduate Studies, Midwestern University, Downers Grove, IL, \\ United States, ${ }^{2}$ Department of Dermatology, Charles C. Gates Center for Regenerative Medicine and Stem Cell Biology, \\ University of Colorado - Anschutz Medical Campus, Aurora, CO, United States, ${ }^{3}$ Institute for Structural Biology, Drug \\ Discovery and Development, Department of Medicinal Chemistry, Virginia Commonwealth University, Richmond, VA, \\ United States
}

Chlamydia is the most frequently reported sexually transmitted bacteria causing 2.9 million infections annually in the United States. Diagnosis, treatment, and sequelae of chlamydial disease cost billions of dollars each year in the United States alone. Considering that a heparin sulfate-like cell surface receptor is involved in Chlamydia infections, we reasoned that sulfated and sulfonated mimics of heparin sulfate would be useful in topical prophylactic prevention of Chlamydia. In this study, we tested a small, synthetic sulfated agent sulfated pentagalloyl glucoside (SPGG) and three synthetic sulfonated polymers PSS and SPS with average molecular weight in the range of 11 to $1000 \mathrm{kDa}$ for inhibition against Chlamydia. Infection of HeLa cells with $C$. muridarum or C. trachomatis in the presence of increasing concentrations of SPGG or sulfonated polymers were quantified by immunofluorescence of Chlamydia inclusions. To determine whether in vitro pre-treatment of SPGG inhibits infection of C. muridarum, HeLa monolayers were incubated with SPGG-containing media, and then infected with Chlamydia. Our in vitro results show that SPGG pre-treatment inhibits Chlamydia infection in a dose-dependent manner. In addition, we further determined if SPGG treatment has an inhibitory effect during infection, therefore cell monolayers were infected with $C$. muridarum in the concurrent presence of SPGG. Our results show that SPGG inhibits $C$. muridarum infection with an $I_{50}$ at $10 \mu \mathrm{g} / \mathrm{ml}$ levels. We also tested the inhibitory effect of synthetic polymers PSS and SPS against Chlamydia and found inhibition of $C$. muridarum and $C$. trachomatis infections with $I_{50}$ ranging from 0.3 to $0.8 \mu \mathrm{g} / \mathrm{ml}$. SPGG, PSS, and SPS inhibit formation of Chlamydia inclusions in a concentration-dependent manner. For evaluation of in vivo efficacy of the most effective agent in blocking C. muridarum, SPGG, we intravaginally pre-treated mice with SPGG before infection with $C$. muridarum. Cervical swabs were collected post-infection to 
quantify Chlamydia inclusions in vitro. Our in vivo data show that the SPGG-treated group has a statistically significant reduction of infection compared to the no-treatment control. Overall, our results show that SPGG could serve as a promising topical inhibitor for preventing Chlamydia infection.

Keywords: Chlamydia trachomatis, Chlamydia muridarum, inhibition, glucoside, SPGG

\section{INTRODUCTION}

Chlamydia sp. are obligate intracellular pathogens and the most common sexually transmitted bacteria worldwide (World Health Organization [WHO], 2016). Human Chlamydia spp. cause several diseases such as cervicitis, trachoma, urethritis, ectopic pregnancy, pelvic inflammatory disease, lymphogranuloma venerum (LGV) as well as others (Häcker, 2018). Chlamydia trachomatis infection is the leading cause of blindness worldwide, while C. muridarum, the murine pathogen closely related to C. trachomatis, is commonly used for modeling human Chlamydial infections in mouse.

Annually, about 20 million people acquire sexually transmitted infections (STI) in the United States. Moreover, in 2016 alone, about 1.5 million cases of C. trachomatis infection were reported to the Centers of Disease Control (Centers for Disease Control and Prevention [CDC], 2017). Although recommended antibiotic treatment for Chlamydia infections is generally effective and antibiotic resistance is thus far rare (Sandoz and Rockey, 2010), most of the infected patients are unlikely to seek treatment. This is because 70 to $90 \%$ of Chlamydia infections in women and $>50 \%$ in men are asymptomatic (Centers for Disease Control and Prevention [CDC], 2017). Untreated Chlamydia infections often cause serious sequelae and complications that lead to infertility (Haggerty et al., 2010; Darville, 2013; Centers for Disease Control and Prevention [CDC], 2017). Furthermore, it has been reported that the standard antibiotic treatment against Chlamydia is less effective than expected possibly due to persistent infections (Kissinger et al., 2016). Although several efforts have been made in public health programs to improve screening and medical treatment to control Chlamydia, the incidence of Chlamydia infection has increased (Rekart and Brunham, 2008; World Health Organization Library [WHO], 2012).

Numerous approaches have been proposed to address this problem; one of which is the use of topical microbicidal or microbistatic prophylaxis. It is believed that new intravaginal compounds that prevent adherence or growth of STI could be effective (Achilles et al., 2002; Stone, 2002; Tiwari et al., 2012; Osaka and Hefty, 2014). Earlier work has shown that heparan sulfate (HS)-like cell surface receptors are involved in mediating Chlamydia infections (Wuppermann et al., 2001; Rosmarin et al., 2012; Tiwari et al., 2012), which has led to the use of new sulfated and sulfonated agents as broadspectrum inhibitors of STI (Herold et al., 1997, 2000; Simoes et al., 2002; Scordi-Bello et al., 2005). Inhibition of Chlamydia spp. infection (C. trachomatis serovar LGV, C. muridarum serovars Nigg) was achieved by exogenous heparin and other negatively charged agents along with cell lines defective in the synthesis of HS (Zaretzky et al., 1995; Wuppermann et al., 2001; Bourne et al., 2003). Although some C. trachomatis serovars (e.g., E and D) were shown to have an infectivity mechanism independent of HS (Taraktchoglou et al., 2001), pretreatment with negatively charged agents are also reported to inhibit these serovars (Zaretzky et al., 1995; Herold et al., 1997, 2000; Achilles et al., 2002).Therefore, we reasoned that agents carrying optimal sulfate or sulfonate groups would potently block microbial attachment and/or reduce host cell-pathogen interaction, thereby reducing Chlamydia infections. This in turn could help reduce transmission of infection and need for antibiotics, possibly reducing incidences of the sequelae of Chlamydia infections.

In this study, we evaluated the anti-Chlamydia activities of a synthetic, small, sulfated agent called sulfated pentagalloyl glucoside (SPGG) with average molecular weight (MW) of $2.2 \mathrm{kDa}$ (structure shown in Supplemental Figure S1), synthetic polymers called poly(sodium 4-styrene sulfonate; PSS) with average MW of $1000 \mathrm{kDa}$, poly(4-styrenesulfonic acid; PSS) with average $\mathrm{MW}$ of $75 \mathrm{kDa}$, and another synthetic polymer called polyanetholsulfonic acid sodium salt (SPS) with average MW of $11 \mathrm{kDa}$. The objective of this study is to test the inhibitory activity of sulfated and sulfonated compounds against Chlamydia. We evaluated the ability of these four different agents to (1) block interaction of host cell with Chlamydia and (2) inhibit Chlamydia growth.

\section{MATERIALS AND METHODS}

\section{Mammalian Cell Lines, Chlamydia Stocks, and Reagents}

HeLa 229 cells (epithelial cervix adenocarcinoma, ATCC CCL2.1) were maintained in DMEM media supplemented with $5 \%$ heat-inactivated fetal bovine serum and $1 \mu \mathrm{g} / \mathrm{ml}$ gentamicin at $37^{\circ} \mathrm{C} 5 \% \mathrm{CO}_{2}$. For these studies, we evaluated C. muridarum Nigg and Weiss strains, as well as C. trachomatis serovar LGV. We obtained all Chlamydia strains from Midwestern University Chlamydia stocks. Initially, C. muridarum Weiss strain was obtained from Dr. T. Cotter who acquired the stocks from laboratory of Dr. H. Caldwell. Dr. H. Caldwell originally obtained this Weiss isolate from J. Schachter, who had obtained it from E. Weiss (Swenson et al., 1983; Ramsey et al., 2009). C. muridarum Nigg strain was originally obtained from Dr. R. Rank of the University of Arkansas who obtained from American Type Culture Collection (Rockville, MD, United States) (Hilleman, 1945). SPGG with average MW of $2.2 \mathrm{kDa}$ was synthesized in the Desai laboratory at Virginia Commonwealth University following earlier reports 
(Al-Horani et al., 2013; Al-Horani and Desai, 2014). PSS with MWs of 75 and $1000 \mathrm{kDa}$ and SPS with a MW of $11 \mathrm{kDa}$ were obtained from Sigma-Aldrich (Catalog \#: 561223, 434574, and 444464, respectively). All reagents were used as received, dissolved or diluted in Milli-Q water for further evaluation. Reagent stocks were prepared at high concentrations (50$100 \mathrm{mg} / \mathrm{ml}$ ) to minimize any effect of the drug diluent (Milli-Q water) after dilution to the working concentrations tested in this study $(\mathrm{ng} / \mathrm{ml}-\mu \mathrm{g} / \mathrm{ml})$. Therefore, untreated control replicates were only treated with media only and diluent was not included in any of these replicates.

\section{Cytotoxicity Assay}

To evaluate the cytotoxicity of the compounds used in this study, we determined the viability of tissue culture cells by 3-(4,5dimethylthiazol-2-yl)-2,5-diphenyltetrazolium bromide (MTT) assay in a 96 well plate. HeLa 229 cells were treated for $24 \mathrm{~h}$ in a humidified $5 \% \quad \mathrm{CO}_{2}$ incubator at $37^{\circ} \mathrm{C}$ with different concentrations of the compounds. After incubation, $10 \mu \mathrm{L}$ of MTT stock solution $(5 \mathrm{mg} / \mathrm{ml}$ ) was added to adherent cells and incubated for four additional hours. Acidic isopropanol $(0.1 \mathrm{~N} \mathrm{HCl}$ in absolute isopropanol) was added after the incubation and absorbance of converted dye was measured at $570 \mathrm{~nm}$ with background subtraction at $630 \mathrm{~nm}$.

\section{Chlamydia in vitro Drug Activity Assays}

For pretreatment of monolayer assays, confluent HeLa 229 cell monolayers were drug treated or untreated for $30 \mathrm{~min}$ at $37^{\circ} \mathrm{C}, 5 \% \mathrm{CO}_{2}$ with different drug concentrations. After the drug treatment, drug containing media was removed and suspension of Chlamydia elementary bodies (EBs) was used to inoculate monolayers in 24-well microtiter plates as described previously (Cotter et al., 1997). Inoculum was rocked for $1 \mathrm{~h}$ at room temperature. Plates were then incubated for two additional hours at $37^{\circ} \mathrm{C}, 5 \% \mathrm{CO}_{2}$. After the incubation period, fresh media containing $1 \mu \mathrm{g} / \mathrm{ml}$ of cycloheximide was added. C. muridarum assays were incubated $24 \mathrm{~h}$ while C. trachomatis assays were incubated for $48 \mathrm{~h}$ at $37^{\circ} \mathrm{C}, 5 \% \mathrm{CO}_{2}$ before plates were fixed with methanol. C. muridarum inclusions were visualized by staining with the $\alpha$-Chlamydia mouse anti-serum paired with secondary FITC-anti mouse antibody (Thermo Fisher Scientific, IL, United States) while C. trachomatis inclusions were stained with commercial biotin-labeled polyclonal $\alpha$ Chlamydia antibody (Fitzgerald Industries International, MA, United States) paired with FITC streptavidin (Invitrogen Corporation, CA, United States). Chlamydia growth was determined by counting fluorescent inclusions viewed through an inverted fluorescence microscope. For pre-treatment of EBs assays, untreated cell monolayers were inoculated with drug treated EBs. Chlamydia EBs were drug treated or untreated for $30 \mathrm{~min}$ at room temperature, centrifuged for $45 \mathrm{~min}$ at $25300 \mathrm{~g}$, drug containing media was removed, and EBs were resuspended in drug-free media before using them as inoculum. For co-treatment, Chlamydia EB inoculum was added simultaneously with drug containing media onto monolayers. Both were rocked for $1 \mathrm{~h}$ at room temperature and then incubated for $2 \mathrm{~h}$ at $37^{\circ} \mathrm{C}, 5 \% \mathrm{CO}_{2}$. After the incubation time, plates were processed as indicated above. Chlamydia infectivity was calculated by counting the inclusion-forming units (IFU) using a Zeiss Axiovert 25 fluorescent microscope to calculate the IFU/ml of each sample. A minimum of 20 fields were counted for each replicate; a minimum of two replicates were evaluated per treatment. The IFU/ml was calculated by multiplying the total number of inclusions obtained per sample by the dilution factor of the sample and by the field factor and divining the final number by the volume of the sample. The field factors vary on the number of fields counted, the area of the wells in each plate and the magnification used. For example for these studies, the field factor for a 24-well plate (Genesee Scientific Corporation, CA, United States) with 20 counted fields at magnification of $40 \times$, has a field factor of 37.26 .

\section{Animal Studies}

Female outbred Swiss Webster mice (35-42 days old and about $20 \mathrm{~g}$ in body weight) were obtained from Charles River Laboratories and used for all experiments. All mice were acclimated to the Animal Resource Facility for at least 7 days prior to inclusion in the experiments. The animal experiments had cohorts that received SPGG topically at different concentrations; in addition, untreated control groups or nodrug treated groups were included. All mice had free access to food and water during the course of these studies and were maintained on a 10:14 (light: dark) cycle. All animal experimental protocols were approved by the Institutional Animal Care and Use Committee. Using a mouse Chlamydia infection model previously described (Ramsey et al., 2001), all mice were treated subcutaneously with $100 \mu \mathrm{L}$ of phosphatebuffered saline (PBS, $0.14 \mathrm{M} \mathrm{NaCl}, 0.01 \mathrm{M}$ phosphate buffer, $\mathrm{pH}$ 7.4) containing $2.5 \mathrm{mg}$ of medroxyprogesterone acetate (Greenstone LLC, NJ, United States) 7 days prior to intravaginal infection. The animal study comprised cohorts that received 30-min topical intravaginal treatment: one cohort received PBS buffer only, one cohort received vehicle gel only and three cohorts received increasing concentrations of SPGG in vehicle gel before Chlamydia infection. The vehicle gel was prepared with $2 \%$ hydroxyethylcellulose (HEC) in PBS. On the day of infection, after the 30-min treatment, all cohorts were inoculated intravaginally with $10 \mu \mathrm{l}$ of C. muridarum Weiss inoculum containing $10^{6}$ IFU in Sucrose - Phosphate Glutamate (SPG) buffer (10 mM phosphate, $0.25 \mathrm{M}$ sucrose, $5 \mathrm{mM}$ L-glutamic acid, $\mathrm{pH}$ 7.4). To evaluate Chlamydia infection from the lower urogenital tract, cervicovaginal swabs with polyester tipped applicators (Puritan ${ }^{\circledR}$ medical products, ME, United States) were collected. Samples were collected at $4,7,10,14$, and 21 days post-infection. All samples were frozen in SPG buffer at $-80^{\circ} \mathrm{C}$ for later batch processing. Chlamydia inclusions were quantified from swabs samples in HeLa 229 cell cultures and enumerated by indirect immunofluorescence as previously described (Cotter et al., 1997). 


\section{Statistical Analysis}

Chlamydia infectivity was calculated by normalizing raw values of inclusion number to percent of the untreated control group or negative control value. In addition, the areas under the treatment time curve (AUC) were calculated for the in vivo experiment data using GraphPad Prism 7 (La Jolla, CA, United States). To determine the effect of the compounds, the concentration of compound where the inhibition is reduced by half $\left(\mathrm{IC}_{50}\right)$ or in $90 \%\left(\mathrm{IC}_{90}\right)$ were determined and different therapies were compared using Student's $t$-test analysis using GraphPad Prism 7 software.

\section{RESULTS}

\section{Activity of Compounds Against Chlamydia}

To determine the anti-Chlamydia activity of the compounds tested in this study, we initially infected HeLa 229 cells with C. muridarum strains Nigg or Weiss or C. trachomatis in the presence of compounds in a dosage dependent manner (co-treatment). Mock treated cells infected with corresponding Chlamydia strain served as a non-treated control (positive control). All four compounds - PSS $1000 \mathrm{kDa}$ (Figures 1A,D,G), PSS $75 \mathrm{kDa}$ (Figures 1B,E,H), SPS $11 \mathrm{kDa}$ (Figures 1C,F,I), and SPGG (Figures 2A-C) - inhibited Chlamydia growth. However, the dose-response inhibition curves were different between the tested compounds (Figures 1, 2 and Table 1).

PSS $1000 \mathrm{kDa}$, PSS $75 \mathrm{kDa}$, and SPS $11 \mathrm{kDa}$ inhibited C. muridarum Nigg strain with $\mathrm{IC}_{50}$ ranging from 0.3 to $0.5 \mu \mathrm{g} / \mathrm{ml}$ while Weiss strain $\mathrm{IC}_{50}$ ranged from 0.4 to $0.8 \mu \mathrm{g} / \mathrm{ml}$ (Figure 1 and Table 1). Inhibition of Chlamydia Nigg strain at $90 \%\left(\mathrm{IC}_{90}\right.$ ) was achieved at concentrations of $1.4 \mu \mathrm{g} / \mathrm{ml}$ PSS $1000 \mathrm{kDa}, 0.9 \mu \mathrm{g} / \mathrm{ml}$ PSS $75 \mathrm{kDa}$, and $8 \mu \mathrm{g} / \mathrm{ml}$ SPS $11 \mathrm{kDa}$,

TABLE 1 | Effects of the sulfated or sulfonated compounds on infection with Chlamydia spp. during co-treatment.

\begin{tabular}{llccc}
\hline $\begin{array}{l}\text { Sulfated or } \\
\text { sulfonated } \\
\text { compound }\end{array}$ & $\begin{array}{l}\text { Chlamydia } \\
\text { strain }\end{array}$ & IC $_{50}(\mu \mathbf{g} / \mathbf{m l})$ & $\begin{array}{c}\mathbf{I C}_{90} \\
(\mu \mathbf{g} / \mathbf{m l})\end{array}$ & $\begin{array}{c}\mathbf{I C}_{50} \mathbf{9 5 \%} \\
\mathbf{C l}^{\#} \\
(\mu \mathbf{g} / \mathbf{m l})\end{array}$ \\
\hline PSS 1000 kDa & Nigg & 0.3 & 1.4 & $0.2-0.4$ \\
& Weiss & 0.6 & 1.9 & $0.3-0.8$ \\
& C. trachomatis & 10 & 42 & $8.7-12$ \\
PSS 75 kDa & Nigg & 0.3 & 0.9 & $0.3-0.4$ \\
& Weiss & 0.8 & 1.4 & $0.5-0.9$ \\
& C. trachomatis & 3.4 & 66 & $1.7-6.7$ \\
SPS 11 kDa & Nigg & 0.5 & 8.0 & $0.3-1.0$ \\
& Weiss & 0.4 & 4.5 & $0.3-0.7$ \\
& C. trachomatis & 7.9 & 13 & $5.5-8.8$ \\
SPGG & Nigg & 1.3 & 230 & $0-3.2$ \\
& Weiss & 2.4 & 12 & $1.3-3.7$ \\
\hline
\end{tabular}

\#Cl: confidence interval. while Weiss strain inhibition was at $1.9,1.4$, and $4.5 \mu \mathrm{g} / \mathrm{ml}$, respectively (Table 1). Inhibition of C. trachomatis was less effective, IC I0 $_{50}$ of PS $1000 \mathrm{kDa}$ was $10 \mu \mathrm{g} / \mathrm{ml}$, PSS $75 \mathrm{kDa}$ was $3.4 \mu \mathrm{g} / \mathrm{ml}$, and SPS $11 \mathrm{kDa}$ was $7.9 \mu \mathrm{g} / \mathrm{ml}$; while $\mathrm{IC}_{90}$ ranged from 13 to $66 \mu \mathrm{g} / \mathrm{ml}$. From all the drugs tested in this study, SPGG is the most effective in blocking C. muridarum. The inhibitory activity of SPGG against C. muridarum Nigg shows an $\mathrm{IC}_{50} 1.3 \mu \mathrm{g} / \mathrm{ml}$ while Weiss strain was $2.4 \mu \mathrm{g} / \mathrm{ml}$ (Figures 2A,B and Table 1). C. trachomatis infectivity was significantly reduced down to $20 \%$ infectivity with $218 \mu \mathrm{g} / \mathrm{ml}$ SPGG (Figure 2C). These results show that all compounds inhibited Chlamydia similarly in the micromolar range. However, these results could not distinguish whether the inhibition resulted from binding to chlamydial ligands or host cell receptors.

\section{Blocking Host Cell Chlamydia Receptors}

In order to determine if PSS $1000 \mathrm{kDa}$, PSS $75 \mathrm{kDa}$, SPS $11 \mathrm{kDa}$ and SPGG were able to block attachment or adhesion of Chlamydia by interacting with host cells, we pre-treated the HeLa 229 monolayers for 30 min with different concentrations of drugs. The excess of drug was removed by pipetting and the wells were washed once with PBS. Then wells were infected with Chlamydia as per standard protocol. Our results show that at $1 \mu \mathrm{g} / \mathrm{ml}$ of PSS $1000 \mathrm{kDa}$, PSS $75 \mathrm{kDa}$, SPS $11 \mathrm{kDa}$, no reduction of C. muridarum Nigg (Figures 3A-C), Weiss (Figures 3D-F) or C. trachomatis (Figures 3G-I) infection was observed. On the other hand, a relatively higher concentration of $100 \mu \mathrm{g} / \mathrm{ml}$ of all three compounds inhibited C. muridarum Nigg to approximately $10 \%$ infectivity while Weiss strain was only reduced by $\sim 60 \%$ infectivity (Figures 3A-F). For C. trachomatis, inhibition was less effective. Some compounds only maintained some residual activity; PSS $1000 \mathrm{kDa}$ and SPS $11 \mathrm{kDa}$ only inhibited $\sim 30 \%$, and PSS $75 \mathrm{kDa} \sim 60 \%$ (Figures 3G-I). Pretreatment of the host cell monolayers with SPGG was effective to reduce $90 \%$ of infectivity of C. muridarum at concentrations of $218 \mu \mathrm{g} / \mathrm{ml}$ (Figures 2D, E), while only $60 \%$ inhibition of C. trachomatis infectivity was observed at similar concentrations (Figure 2F). These results showed the assessed compounds reduce attachment of C. muridarum and C. trachomatis to host cell only at $100 \mu \mathrm{g} / \mathrm{ml}$ PSS $1000 \mathrm{kDa}$, PSS $75 \mathrm{kDa}$, SPS $11 \mathrm{kDa}$ and $43.6 \mu \mathrm{g} / \mathrm{ml}$ SPGG; and it is less effective to reduce $C$. trachomatis than C. muridarum. Therefore, the compounds might be working at the point of attachment blocking interaction of Chlamydia with the eukaryotic cell surface receptor but pre-treatment of the host cell is not as effective as when the compounds are present together with chlamydial EBs at the time of infection.

\section{Blocking Chlamydia EBs}

To determine whether chlamydial inhibition resulted from binding of the compounds to chlamydial EB ligands, we pretreated Chlamydia EBs for $30 \mathrm{~min}$ with $100 \mu \mathrm{g} / \mathrm{ml}$ of sulfated or sulfonated compounds, removed the drug by centrifugation and then used those EBs to challenge host cell monolayers. Our results show that the pre-treatment of EBs reduced the infectivity of the Chlamydia. C. muridarum Nigg strain was 


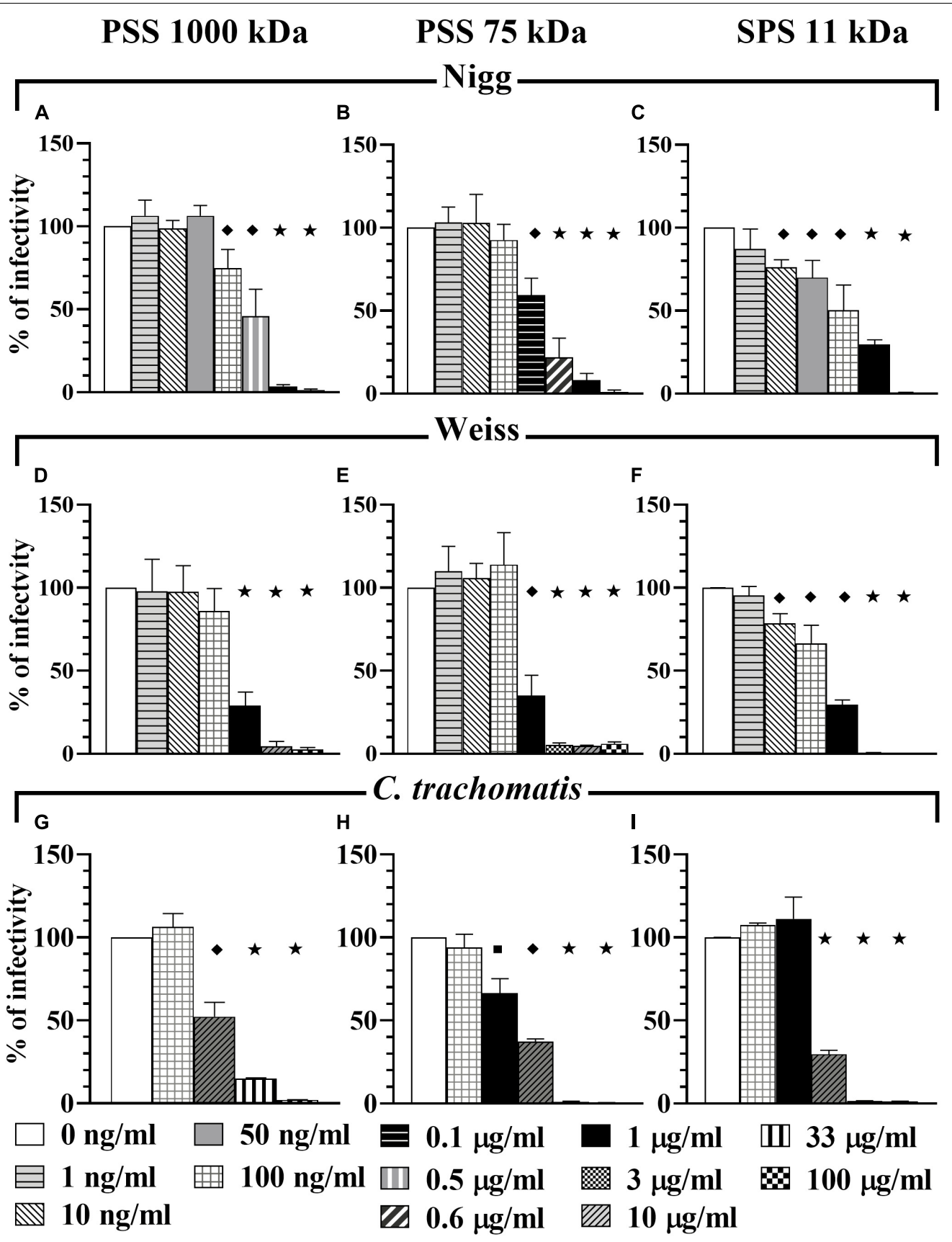

FIGURE 1 | Co-treatment activity of PSS $1000 \mathrm{kDa}$, PSS $75 \mathrm{kDa}$, and SPS $11 \mathrm{kDa}$ against C. muridarum Nigg and Weiss strains and C. trachomatis. C. muridarum EBs Nigg (A-C), Weiss strain (D-F), and C. trachomatis (G-I) were used to infect HeLa cells in the presence of different drug concentrations (co-treatment). Multiplicity of infection (MOI) used in this in vitro study was 1. After infection, plates were fixed and IFU were detected by immunofluorescence and counted as indicated in Materials and Methods. The inclusion values of all treatments were normalized to the non-treated control as percentage (\% of infectivity). Bars represent the mean per treatment of at least three independent experiments; error bars indicate the standard deviation (SD). Stars ( $\star$ ) indicate statistical significance when compare to the non-treated control with a $p<0.0001$, diamonds ( $)$ indicate statistical significance with a $p<0.008$, and squares $(\square)$ indicate a $p \leq 0.03$ as calculated by Student's $t$-test.

significantly reduced in infectivity by $80 \%$ with PSS $1000 \mathrm{kDa}$, $60 \%$ with both PSS $75 \mathrm{kDa}$ and SPS $11 \mathrm{kDa}$, and $80 \%$ with SPGG (Figure 4A). Similarly, C. muridarum Weiss infectivity was reduced by $80,50,40$, and $50 \%$, respectively. C. trachomatis had a reduction of $70 \%$ infectivity with all polymer agents
(PSS $1000 \mathrm{kDa}$, PSS $75 \mathrm{kDa}$, and SPS $11 \mathrm{kDa}$ ) and 40\% with SPGG. These results show that the sulfated compounds interact with both the eukaryotic host cells (Figures 2D-F, 3) and Chlamydia EBs (Figure 4) to block or reduce infection. These experiments indicate that the compounds are likely acting at 


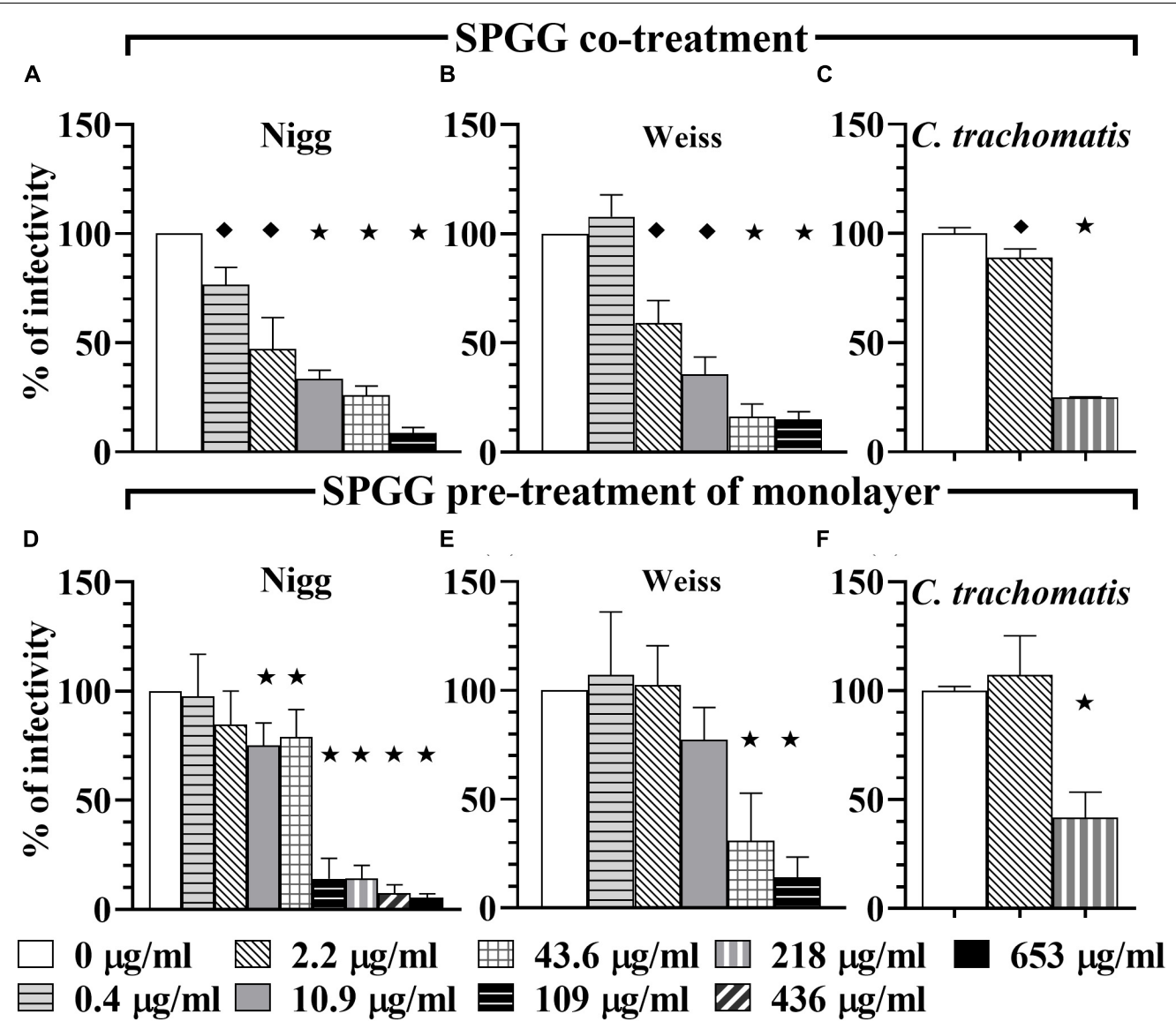

FIGURE 2 | Activity of SPGG against C. muridarum (Nigg and Weiss) and C. trachomatis. EBs of C. muridarum Nigg (A,D), Weiss strain (B,E) and C. trachomatis $(\mathbf{C}, \mathbf{F})$ were used to infect HeLa cells in the presence of different drug concentrations (co-treatment, panels $\mathbf{A}-\mathbf{C}$ ) or monolayers were drug-treated before infection (pre-treatment of monolayers: D-F). MOI used in this in vitro study was 1. After infection, plates were fixed and IFU were detected by immunofluorescence and counted as indicated in Materials and Methods. The inclusion values of all treatments were normalized to the non-treated control as percentage (\% of infectivity). Bars represent the mean per treatment of at least three independent experiments, error bars indicate the SD. Stars ( $\star$ ) indicate statistical significance when compare to the non-treated control with a $p<0.0001$, diamonds $(\checkmark)$ indicate statistical significance with a $p \leq 0.007$, and squares $(\mathbf{\square})$ indicate a $p \leq 0.01$ as calculated by Student's $t$-test.

the point of attachment of Chlamydia by interacting with both Chlamydia cell surface molecules and eukaryotic cell surface receptors.

\section{Cytotoxicity}

To assess the potential cytotoxicity of the compounds for HeLa cells, cell proliferation and viability was determined as the conversion of water soluble MTT to a purple insoluble formazan product. PSS $75 \mathrm{kDa}$, and SPS $11 \mathrm{kDa}$ did not display any cytotoxicity for HeLa cells at concentrations up to $1000 \mu \mathrm{g} / \mathrm{ml}$, whereas PSS $1000 \mathrm{kDa}$ reduced cell viability $\sim 25 \%$ at $1000 \mu \mathrm{g} / \mathrm{ml}$. Similarly, SPGG did not exhibit any cytotoxicity at concentration up to $218 \mu \mathrm{g} / \mathrm{ml}$ (Supplemental Figure S2).

\section{Efficacy of SPGG in the Mouse Intravaginal Infection Model}

Finally, the most effective in vitro compound, SPGG against C. muridarum infection, was tested to evaluate its efficacy in vivo. To further explore its potential as an anti-Chlamydia therapy, SPGG was used as a topical pre-treatment in a mouse model. Female Swiss Webster mice were treated intravaginally for $30 \mathrm{~min}$ with 50,500, and $4000 \mu \mathrm{g} / \mathrm{ml}$ of SPGG, SPG buffer only or vehicle only. No vaginal irritation or mouse distress was observed following intravaginal administration of single dose. Microbial burden was monitored by quantification of Chlamydia content in intravaginal swabs after in vitro infection and immunofluorescence staining.

In our in vivo experiments, all animals of the buffer-onlytreated mice became infected and almost all (9/10) animals that received vehicle gel became infected. These data are consistent with no significant protective effect of the buffer or placebo gel. In contrast, while all animals became infected, SPGG gel shows some protective efficacy against Chlamydia infection in this model by reducing the microbial burden in $50 \%$ or more (Figure 5A). The AUC values of each SPGG-treated cohort (Figures 5B: G3, G4, G5) were compared to the nontreated control value (Figure 5B: G1) using a Student's $t$-test. 

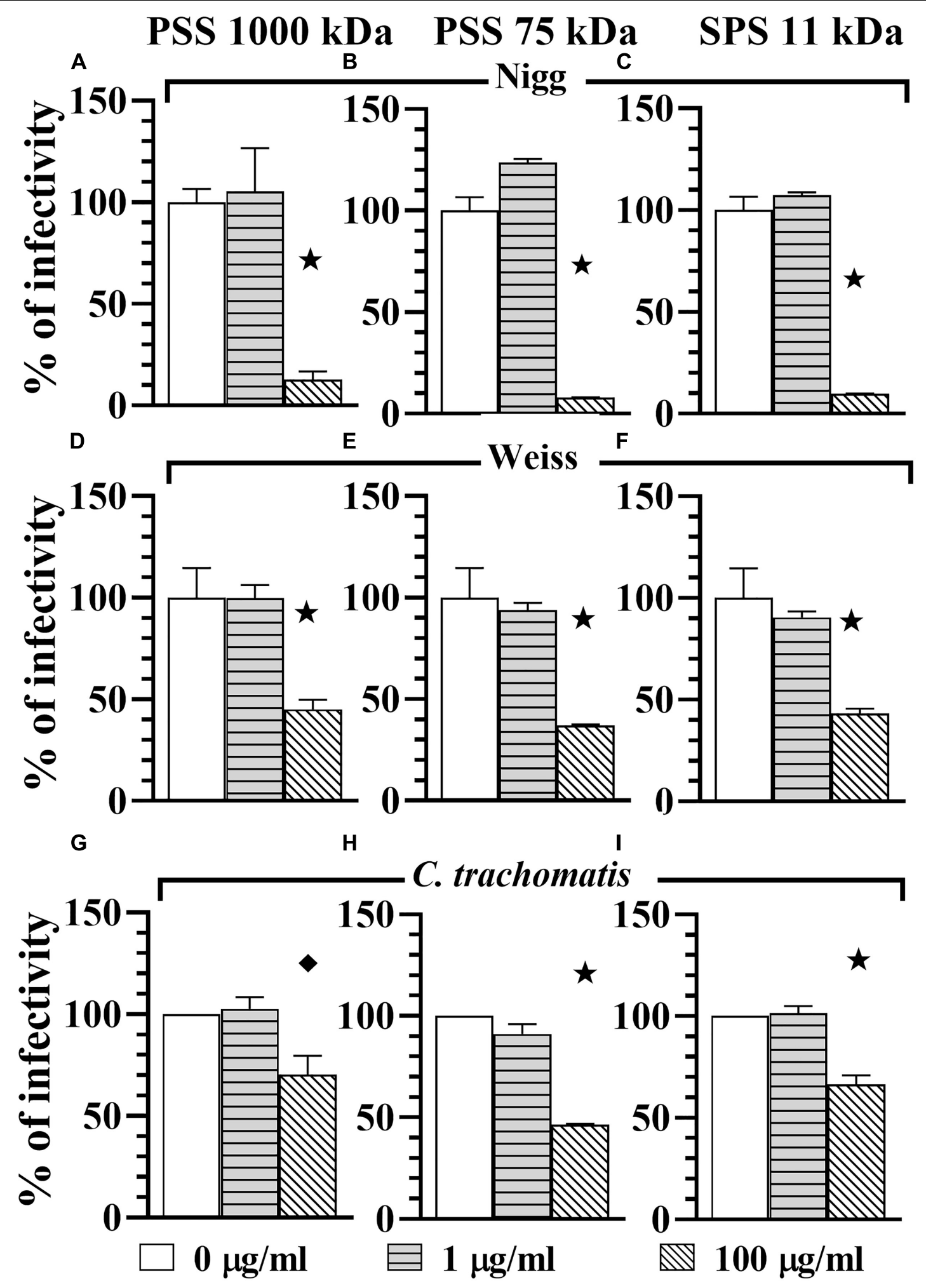

FIGURE 3 | Host-cell blocking activity of PSS 1000 kDa, PSS 75 kDa, and SPS 11 kDa against C. muridarum Nigg and Weiss strains and C. trachomatis. Chlamydia EBs Nigg (A-C), Weiss strain (D-F), and C. trachomatis (G-I) were used to infect HeLa cells. Monolayers were drug-treated before infection (pre-treatment of monolayers). MOI used in this in vitro study was 1. After infection, plates were fixed and IFU were detected by immunofluorescence and counted as indicated in Materials and Methods. The inclusion values of all treatments were normalized to the non-treated control as percentage (\% of infectivity). Bars represent the mean per treatment of two independent experiments, error bars indicate the SD. Stars ( $\star$ ) indicate statistical significance when compare to the non-treated control with a $p<0.0001$, and diamonds $(\bullet)$ indicate statistical significance with a $p \leq 0.007$ as calculated by Student's $t$-test. 


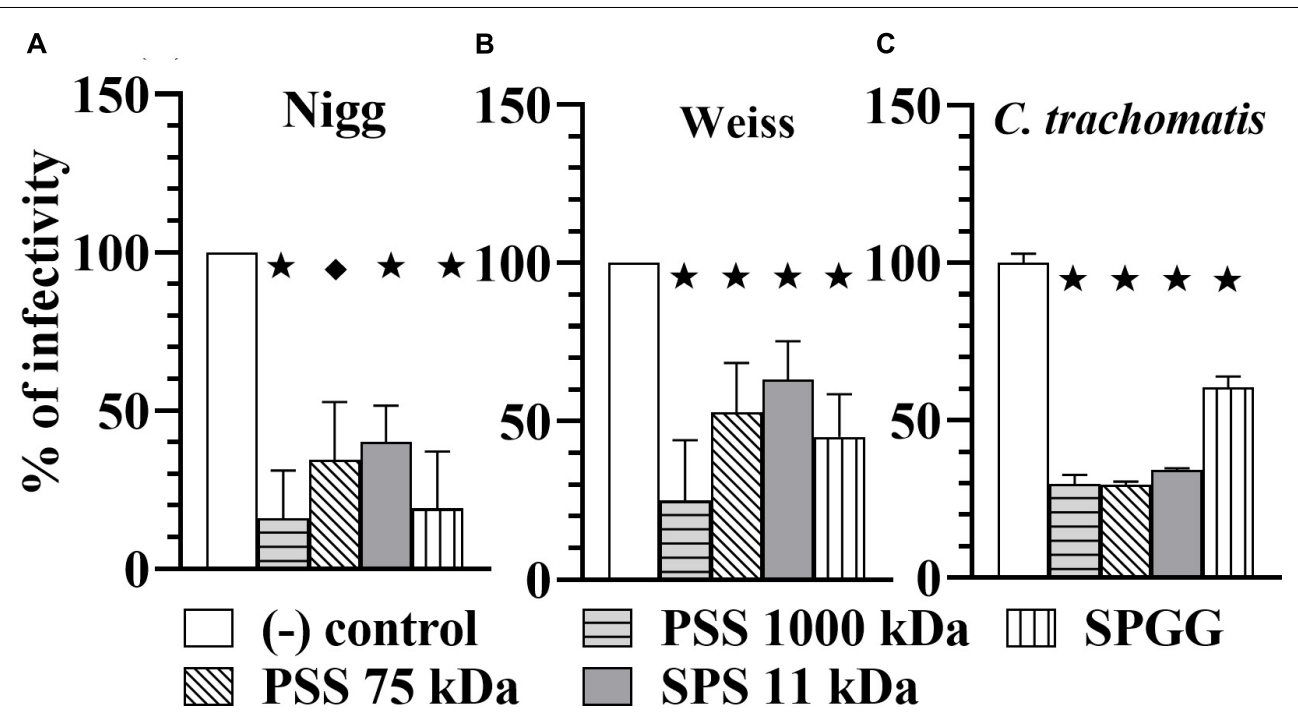

FIGURE 4 | Microbial-blocking activity of PSS 1000 kDa, PSS 75 kDa, SPS 11 kDa and SPGG against C. muridarum Nigg (A), Weiss (B) strains and C. trachomatis (C). Chlamdyia EBs were drug-treated with $100 \mu \mathrm{g} / \mathrm{ml}$ of PSS $1000 \mathrm{kDa}$, PSS $75 \mathrm{kDa}$, SPS $11 \mathrm{kDa}$, or $218 \mu \mathrm{g} / \mathrm{ml}$ SPGG before infecting HeLa cells. MOI used in this in vitro study was 3. After infection, plates were fixed and IFU were detected by immunofluorescence and counted as indicated in Materials and Methods. The inclusion values of all treatments were normalized to the non-treated control as percentage (\% of infectivity). Bars represent the mean per treatment of at least two independent experiments, error bars indicate the SD. Stars $(\star)$ indicate statistical significance when compare to the non-treated control with a $p<0.0001$, and diamonds $(\checkmark)$ indicate statistical significance with a $p \leq 0.007$ as calculated by Student's $t$-test.
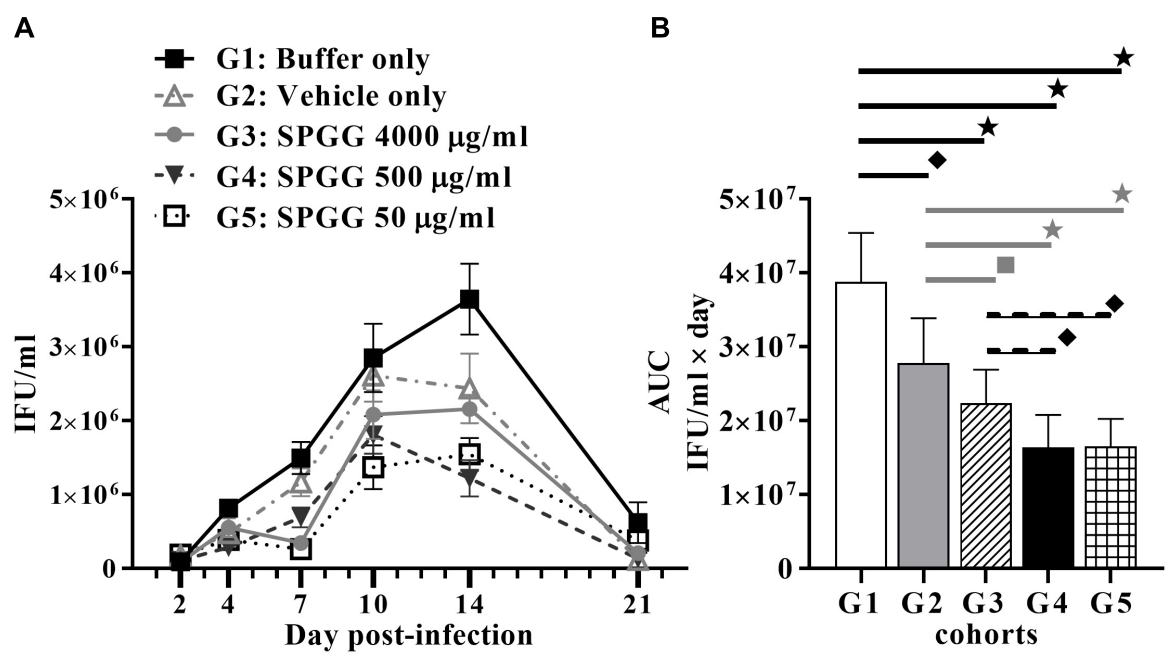

FIGURE 5 | Anti-Chlamydia activity of SPGG in mice. (A) Swiss Webster Mice were topically treated with SPGG before intravaginal infection with C. muridarum Weiss strain. Infection was monitored by intravaginal swabbing on day 2, 4, 7, 10, 14, and 21 post-infection and measuring the Chlamydia growth in tissue culture as described in Materials and Methods. Plots represent the mean per treatment of at least ten animals per group, error bars indicate the SD. (B) Statistical analysis of the AUC of anti-Chlamydia activity of SPGG in mice. AUCs were calculated as indicated in Materials and Methods. G1: Buffer only, G2: Vehicle only, G3: SPGG $4000 \mu \mathrm{g} / \mathrm{ml}$, G4: SPGG $500 \mu \mathrm{g} / \mathrm{ml}$, and G5: SPGG $50 \mu \mathrm{g} / \mathrm{ml}$. Horizontal lines indicate the cohorts compared with statistically significant differences. Stars ( $\star$ ) indicate statistical significance of compared cohorts with a $p \leq 0.0002$, diamonds $(\boldsymbol{})$ indicate statistical significance with a $p \leq 0.0036$, and squares $(\boldsymbol{\square})$ indicate a $p \leq 0.021$ as calculated by Student's $t$-test.

This statistical analysis demonstrated that there is significant difference in all drug-treated cohorts compared ( $p \leq 0.0001)$. In addition, an ordinary one-way Analysis of Variance (ANOVA) $t$-test was performed comparing all pairs of cohorts resulting in a significant difference of all cohorts $(p \leq 0.0001)$. Even though, we observed a hindering effect of vehicle-only cohort in Chlamydia infectivity (Figures 5A,B: G2), when the three SPGG-treated cohorts were compared individually to vehicle-only cohort a significant difference $(p \leq 0.0145)$ was observed using a Student's t-test. In addition, when SPGG-treated cohorts (Figures 5B: G3-G5) were compared between each other, there was significant statically difference ( $p \leq 0.0145$ ); however, treatment did not 
display a sigmoidal dose-response curve (Figure 5B). Taken all together, these results demonstrate that a topical gel containing SPGG can reduce microbial burden of Chlamydia.

\section{DISCUSSION}

Chlamydia is a significant public health problem in the United States and throughout the globe; costing millions of dollars each year. The majority of infected patients, both women and men, are asymptomatic. The lack of treatment causes severe sequelae that prominently include infertility and blindness, making the Chlamydia sp. the most common cause of infertility in women and the world's leading cause of microbial blindness (Centers for Disease Control and Prevention [CDC], 2017). An antimicrobial therapy that prevents chlamydial attachment or reduces infectious burden has the potential to decrease transmissibility or reduce clinical manifestations associated with this infection. However, most therapies available do not prevent Chlamydia attachment before infection is established. Thus, there is a need for novel therapeutic strategies to prevent Chlamydia infection. The main objective of this study was to compare the effectiveness of Chlamydia inhibition of four sulfonated and sulfated agents, PSS $1000 \mathrm{kDa}$, PSS $75 \mathrm{kDa}$, SPS $11 \mathrm{kDa}$, and SPGG as monotherapy against Chlamydia, in an attempt to identify a potential compound to prevent or reduce infection. These four compounds, or a derivative thereof, are attractive candidates to treat Chlamydia infections because they have been reported to have broad spectrum antimicrobial activity against other pathogens such as Gardnerella sp., Bacteriodes sp. (Simoes et al., 2002), herpes virus (Gangji et al., 2018) and HIV (Nakashima et al., 1996).

This study has shown that each polyanionic agent inhibits chlamydial infection in an in vitro model. Although each of the agents tested blocked chlamydial infection, SPGG was the most effective in blocking C. muridarum in this study. Sulfated polyanions that block Chlamydia infection are commonly reported to have multiple sulfate groups that attempt to compete with the sulfated glycosaminoglycan chains present on cell surfaces. Although this is a possible mechanism of inhibition, the precise mode of action of these negatively charged compounds remains to be fully understood. Some reports showed that it depends on the number of charged groups available for interaction with both eukaryotic host cell membrane proteins and microbial surface proteins (Herold et al., 2000; Scordi-Bello et al., 2005). Molecules with several negatively charged groups seem to be more efficient in reducing or blocking infection. On the other hand, the total MW of the compound does not play a substantial role in the inhibition. In this study, our results confirm those findings. The structure of PSS $1000 \mathrm{kDa}$, PSS $75 \mathrm{kDa}$, SPS $11 \mathrm{kDa}$ show that each one had a single negatively charged group per monomer and they mainly differ in their MW (Supplemental Figure S1). Our results show that all three compounds had very little difference in $\mathrm{IC}_{50}$ values and percent of inhibition in all inhibition studies: co- and pre-treatment of monolayer or
Chlamydia EBs. In addition, the SPGG structure shows that it has several sulfated groups per molecule and yet only $2.2 \mathrm{kDa}$ MW. It is a highly negatively charged, synthetic small molecule and therefore carries the most promise in this study. Although there was no correlation with MW and efficacy of the compound used, there was a correlation with the degree of sulfation present on the anti-Chlamydia agent.

Equally important, SPGG is a highly soluble glucoside that does not have cytotoxic effects on human cervical epithelial tissue culture cells (Supplemental Figure S2). SPGG is also reported to be effective against other STDs such as herpes virus 1 (Gangji et al., 2018) and HIV (Nakashima et al., 1996). Thus, SPGG or similar derivatives deserve further development, more elaborate animal model testing and clinical evaluation. Here, our data illustrate that SPGG inhibits both mouse pathogen C. muridarum in vitro and in vivo and human pathogen C. trachomatis.

Even though promising results are reported here, there are certainly limitations to our study. Our antimicrobial screening was conducted as monotherapy with only two C. muridarum strains and one C. trachomatis LGV strain. Future studies should evaluate the inhibitory activity of these sulfated agents against other clinically relevant isolates, other Chlamydia spp. serovars (e.g., A, E, or D), and in other animal models using a monotherapy or combination therapy. Another potential weakness was the dose of $C$. muridarum used in the mouse. Our group typically uses a Chlamydia dose of $\sim 10^{4}$ IFU to infect BALB/c or C57BL/6J mice, whereas in this study, a $2 \log _{10}$ higher challenge $\left(10^{6} \mathrm{IFU}\right)$ was used to infect Swiss Webster mice that required a higher dose to achieve $100 \%$ infection. This higher dose may be the reason why, though we observed a reduced infectious burden with doses of SPGG, we did not observe a sigmoid curve or absolute prevention of infection at any concomitantly increasing dose. To further investigate this effect and clarify the inhibitory role on SPGG in vivo, new studies should be performed with different mouse strains and several inoculum doses. Also, considering that infectivity of Chlamydia sp. serovar LGV strongly depends on a HS-related mechanism; while other urogenital Chlamydia serovars (e.g., E) hinge less on HS-like GAGs, a comparison of infection of several Chlamydia serovars should be performed to fully understand the inhibitory role of these polyanion compounds. Other animal infection models should also be included in future studies such as the upper urinary tract infection or the trachoma infection model. Finally, we believe the observed hindering effect of the vehicle gel $(2 \%$ hydroxylcellulose) should be explored in detail to optimize the effect of the polyanion agents, along with the effect of different gel vehicles for drug delivery.

\section{CONCLUSION}

In conclusion, our results show that SPGG had good inhibitory activity against two C. muridarum strains and one C. trachomatis LGV strain. This study provides evidence that SPGG topical prophylaxis reduced Chlamydia infection and may serve as a potential therapeutic strategy to prevent Chlamydia growth of serovars with a GAG-dependent mechanism for infection. 


\section{ETHICS STATEMENT}

This study was carried out in accordance with the recommendations described in the Public Health Service Policy on Humane Care and Use of Laboratory Animals and the Guide for the Care and Use of Laboratory Animals. The MWU animal protocol "Evaluation of sexual transmission of Chlamydia in the mouse" was approved by the MWU Institutional Animal Care and Use Committee (IACUC).

\section{AUTHOR CONTRIBUTIONS}

KG directed the project, conceived, and designed the experiments with supervision of KR. KG, CT, DR, RDT, DG, and KR performed the experiments. KG, CT, DR, RDT, and DJ contributed in data acquisition. KG analyzed and interpreted the data. VT and UD contributed reagents. KG wrote the manuscript with support from KR. CT proofread the manuscript.

\section{FUNDING}

This work was supported by intramural funding of Midwestern University. The funders had no role in study design, data collection and analysis, decision to publish, or preparation of the

\section{REFERENCES}

Achilles, S. L., Shete, P. B., Whaley, K. J., Moench, T. R., and Cone, R. A. (2002). Microbicide efficacy and toxicity tests in a mouse model for vaginal transmission of Chlamydia trachomatis. Sex. Transm. Dis. 29, 655-664. doi: 10.1097/00007435-200211000-00007

Al-Horani, R. A., and Desai, U. R. (2014). Designing allosteric inhibitors of factor XIa. lessons from the interactions of sulfated pentagalloylglucopyranosides. J. Med. Chem. 57, 4805-4818. doi: 10.1021/jm500311e

Al-Horani, R. A., Ponnusamy, P., Mehta, A. Y., Gailani, D., and Desai, U. R. (2013). Sulfated pentagalloylglucoside is a potent, allosteric, and selective inhibitor of factor XIa. J. Med. Chem. 56, 867-878. doi: 10.1021/jm301338q

Bourne, N., Zaneveld, L. J. D., Ward, J. A., Ireland, J. P., and Stanberry, L. R. (2003). Poly(sodium 4-styrene sulfonate): evaluation of a topical microbicide gel against herpes simplex virus type 2 and Chlamydia trachomatis infections in mice. Clin. Microbiol. Infect. 9, 816-822. doi: 10.1046/j.1469-0691.2003. 00659.x

Centers for Disease Control and Prevention [CDC] (ed.) (2017). Sexually Transmitted Disease Surveillance 2016. Atlanta: U.S. Department of Health and Human Services.

Cotter, T. W., Ramsey, K. H., Miranpuri, G. S., Poulsen, C. E., and Byrne, G. I. (1997). Dissemination of Chlamydia trachomatis chronic genital tract infection in gamma interferon gene knockout mice. Infect. Immun. 65, 2145-2152.

Darville, T. (2013). "Recognition and treatment of chlamydial infections from birth to adolescence," in Hot Topics in Infection and Immunity in Children IX, eds N. Curtis, A. Finn, and A. J. Pollard (Berlin: Springer), 109-122.

Gangji, R. N., Sankaranarayanan, N. V., Elste, J., Al-Horani, R. A., Afosah, D. K., Joshi, R., et al. (2018). Inhibition of herpes simplex Virus-1 entry into human cells by nonsaccharide glycosaminoglycan mimetics. ACS Med. Chem. Lett. 9, 797-802. doi: 10.1021/acsmedchemlett.7b00364

Häcker, G. (2018). Biology of Chlamydia. New York, NY: Springer. doi: 10.1007/ 978-3-319-71232-1

Haggerty, C. L., Gottlieb, S. L., Taylor, B. D., Low, N., Xu, F., and Ness, R. B. (2010). Risk of sequelae after Chlamydia trachomatis genital infection in women. J. Infect. Dis. 201(Suppl. 2), S134-S155. doi: 10.1086/652395 manuscript. The synthesis of SPGG was funded by grant from the NHLBI R01 HL090586 to UD.

\section{ACKNOWLEDGMENTS}

We want to express our special thanks to Dr. Rafael Masitas at Florida State University for his help with the drawing of the chemical structures. We also want to thank Dr. I. Sigar at Midwestern University for her feedback on this project.

\section{SUPPLEMENTARY MATERIAL}

The Supplementary Material for this article can be found online at: https://www.frontiersin.org/articles/10.3389/fmicb. 2018.03269/full\#supplementary-material

FIGURE S1 | Chemical structures of sulfated and sulfonated compounds tested in this study.

FIGURE S2 | Cytotoxicity of HeLa cells after $24 \mathrm{~h}$ exposure with PSS $1000 \mathrm{kDa}$, PSS 75 kDa, SPS 11 kDa, and SPGG. MTT assay was performed as indicated in Materials and Methods. OD values were normalized to percent of the non-treated control. Bars represent the mean per treatment of at least two independent experiments, error bars indicate the SD. Stars $(\star)$ indicate statistical significance when compare to the non-treated control with a $p<0.0001$, and diamonds $(\checkmark)$ indicate statistical significance with a $p<0.005$ as calculated by Student's $t$-test.

Herold, B. C., Bourne, N., Marcellino, D., Kirkpatrick, R., Strauss, D. M., Zaneveld, L. J. D., et al. (2000). Poly(sodium 4-styrene sulfonate): an effective candidate topical antimicrobial for the prevention of sexually transmitted diseases. J. Infect. Dis. 181, 770-773. doi: 10.1086/315228

Herold, B. C., Siston, A., Bremer, J., Kirkpatrick, R., Wilbanks, G., Fugedi, P., et al. (1997). Sulfated carbohydrate compounds prevent microbial adherence by sexually transmitted disease pathogens. Antimicrob. Agents Chemother. 41, 2776-2780. doi: 10.1128/AAC.41.12.2776

Hilleman, M. R. (1945). Immunological studies on the psittacosislymphogranuloma group of viral agents. J. Infect. Dis. 76, 96-114. doi: 10.1093/infdis/76.2.96

Kissinger, P. J., White, S., Manhart, L. E., Schwebke, J., Taylor, S. N., Mena, L., et al. (2016). Azithromycin treatment failure for chlamydia trachomatis among heterosexual men with nongonococcal urethritis. Sex. Transm. Dis. 43, 599-602. doi: 10.1097/OLQ.0000000000000489

Nakashima, H., Ichiyama, K., Hirayama, F., Uchino, K., Ito, M., Saitoh, T., et al. (1996). Sulfated pentagalloyl glucose (Y-ART-3) inhibits HIV replication and cytopathic effects in vitro, and reduces HIV infection in hu-PBL-SCID mice. Antiviral Res. 30, 95-108. doi: 10.1016/0166-3542(95) 00903-5

Osaka, I., and Hefty, P. S. (2014). Lipopolysaccharide-binding alkylpolyamine DS-96 inhibits Chlamydia trachomatis infection by blocking attachment and entry. Antimicrob. Agents Chemother. 58, 3245-3254. doi: 10.1128/aac.023 91-14

Ramsey, K. H., Miranpuri, G. S., Sigar, I. M., Ouellette, S., and Byrne, G. I. (2001). Chlamydia trachomatis persistence in the female mouse genital tract: inducible nitric oxide synthase and infection outcome. Infect. Immun. 69, 5131-5137. doi: 10.1128/iai.69.8.5131-5137.2001

Ramsey, K. H., Sigar, I. M., Schripsema, J. H., Denman, C. J., Bowlin, A. K., Myers, G. A. S., et al. (2009). Strain and virulence diversity in the mouse pathogen Chlamydia muridarum. Infect. Immun. 77, 3284-3293. doi: 10.1128/iai.00 147-09

Rekart, M. L., and Brunham, R. C. (2008). Epidemiology of chlamydial infection: are we losing ground? Sex. Transm. Infect. 84, 87-91. doi: 10.1136/sti.2007. 027938 
Rosmarin, D. M., Carette, J. E., Olive, A. J., Starnbach, M. N., Brummelkamp, T. R., and Ploegh, H. L. (2012). Attachment of Chlamydia trachomatis L2 to host cells requires sulfation. Proc. Natl. Acad. Sci. 109, 10059-10064. doi: 10.1073/pnas. 1120244109

Sandoz, K. M., and Rockey, D. D. (2010). Antibiotic resistance in chlamydiae. Fut. Microbiol. 5, 1427-1442. doi: 10.2217/fmb.10.96

Scordi-Bello, I. A., Mosoian, A., He, C., Chen, Y., Cheng, Y., Jarvis, G. A., et al. (2005). Candidate sulfonated and sulfated topical microbicides: comparison of anti-human immunodeficiency virus activities and mechanisms of action. Antimicrob. Agents Chemother. 49, 3607-3615. doi: 10.1128/aac.49.9.36073615.2005

Simoes, J. A., Citron, D. M., Aroutcheva, A., Anderson, R. A., Chany, C. J. II, Waller, D. P., et al. (2002). Two novel vaginal microbicides (polystyrene sulfonate and cellulose sulfate) inhibit Gardnerella vaginalis and anaerobes commonly associated with bacterial vaginosis. Antimicrob. Agents Chemother. 46, 2692-2695. doi: 10.1128/aac.46.8.2692-2695.2002

Stone, A. (2002). Microbicides: a new approach to preventing HIV and other sexually transmitted infections. Nat. Rev. Drug Discov. 1:977. doi: 10.1038/ nrd959

Swenson, C. E., Donegan, E., and Schachter, J. (1983). Chlamydia trachomatisinduced salpingitis in mice. J. Infect. Dis. 148, 1101-1107. doi: 10.1093/infdis/ 148.6.1101

Taraktchoglou, M., Pacey, A. A., Turnbull, J. E., and Eley, A. (2001). Infectivity of Chlamydia trachomatis serovar LGV but not $\mathrm{E}$ is dependent on host cell heparan sulfate. Infect. Immun. 69, 968-976. doi: 10.1128/IAI.69.2.968-976. 2001
Tiwari, V., Maus, E., Sigar, I. M., Ramsey, K. H., and Shukla, D. (2012). Role of heparan sulfate in sexually transmitted infections. Glycobiology 22, 1402-1412. doi: $10.1093 /$ glycob/cws106

World Health Organization [WHO] (2016). WHO Guidelines for the Treatment of Chlamydia trachomatis. Geneva: World Health Organization.

World Health Organization Library [WHO] (ed.) (2012). Global Incidence and Prevalence of Selected Curable Sexually Transmitted Infections - 2008. Geneva: World Health Organization Press.

Wuppermann, F. N., Hegemann, J. H., and Jantos, C. A. (2001). Heparan sulfatelike glycosaminoglycan is a cellular receptor for Chlamydia pneumoniae. J. Infect. Dis. 184, 181-187. doi: 10.1086/322009

Zaretzky, F. R., Pearce-Pratt, R., and Phillips, D. M. (1995). Sulfated polyanions block Chlamydia trachomatis infection of cervix-derived human epithelia. Infect. Immun. 63, 3520-3526.

Conflict of Interest Statement: The authors declare that the research was conducted in the absence of any commercial or financial relationships that could be construed as a potential conflict of interest.

Copyright (C) 2019 Gallegos, Taylor, Rabulinski, Del Toro, Girgis, Jourha, Tiwari, Desai and Ramsey. This is an open-access article distributed under the terms of the Creative Commons Attribution License (CC BY). The use, distribution or reproduction in other forums is permitted, provided the original author(s) and the copyright owner(s) are credited and that the original publication in this journal is cited, in accordance with accepted academic practice. No use, distribution or reproduction is permitted which does not comply with these terms. 